\title{
EIGENVALUES OF THE KLEIN-GORDON EQUATION
}

\author{
$b y$ BRANKO NAJMAN†
}

(Received 25th August 1981)

Consider the Klein-Gordon equation

$$
\left[\left(\frac{\partial}{\partial t}-i e q\right)^{2}-\sum_{j=1}^{n}\left(\frac{\partial}{\partial x_{j}}-i e A_{j}\right)^{2}+m^{2}\right] u=0
$$

where $q, A_{j}$ are real valued functions on $R^{n}, m$ and $e$ positive constants. Equation (1) describes the motion of a relativistic particle of mass $m$ and charge $e$ in an external field described by the electrostatic potential $q$ and the electromagnetic potential $A=\left(A_{j}\right)$; units are chosen so that the speed of light is one.

Assume

$$
q \in L^{\infty}\left(R^{n}\right), \quad A_{j} \in L_{\text {loc }}^{2}\left(R^{n}\right)
$$

Denote by $K$ the operator of multiplication by $e q$ in $\mathscr{G}=L^{2}\left(R^{n}\right) ; K$ is a bounded selfadjoint operator. $\tilde{H}$ is the natural selfadjoint realisation of

$$
-\sum_{j=1}^{n}\left(\frac{\partial}{\partial x_{j}}-i e A_{j}\right)^{2}+m^{2} \text { in } \mathscr{G}([3]), H=\tilde{H}-K^{2}
$$

The equation (1) can be written as

$$
\frac{d^{2} u(t)}{d t^{2}}-2 i K \frac{d u(t)}{d t}+H u(t)=0
$$

or, using the notation

$$
U(t)=\left[\begin{array}{c}
u(t) \\
-i \frac{d u(t)}{d t}
\end{array}\right], \quad \mathscr{A}=\left[\begin{array}{cc}
0 & \mathrm{I} \\
\mathrm{H} & 2 \mathrm{~K}
\end{array}\right]
$$

we have

$$
\frac{d U(t)}{d t}=i \mathscr{A} U(t)
$$

$†$ This research was done while the author was visiting the Department of Mathematics, University of California, Berkeley, with the support of a Fulbright grant and SIZ VI SRH. 
It is easy to see that (3) has an elementary solution of the form $u(t)=e^{i \lambda t} u, u \in \mathscr{G}$, if and only if (4) has a solution of the form

$$
U(t)=e^{i \lambda t}\left[\begin{array}{l}
u \\
\lambda u
\end{array}\right]
$$

This is the case if and only if $\lambda$ is an eigenvalue of $\mathscr{A}$; equivalently if and only if

$$
\left(\lambda^{2}-2 \lambda K-H\right) u=0
$$

We say that $\lambda$ is an eigenvalue, $u$ an eigenfunction of the Klein-Gordon equation if (5) holds. It is an eigenvalue of geometric (algebraic) multiplicity $k$ if it is an eigenvalue of $\mathscr{A}$ of geometric (algebraic) multiplicity $k$; here the geometric (algebraic) multiplicity of an eigenvalue of a linear operator is the dimension of the eigenspace (of the space spanned by eigenvectors and the associated vectors). The eigenvalue is simple if it is of algebraic multiplicity one and it is semisimple if its algebraic and geometric multiplicities are equal. If $\lambda$ is a nonsemisimple eigenvalue, then there is an eigenfunction $u$ and a function $v$ such that $\left(\lambda^{2}-2 \lambda K-H\right) v=$ $2(\lambda-K) u$.

If $H$ is positive definite all the eigenvalues are real and semisimple (this follows from the fact that $\mathscr{A}$ is selfadjoint in the Hilbert space to be defined below). In general, there might be nonreal and nonsemisimple eigenvalues; this is the so-called Klein paradox. It cannot happen as long as $\|K\|<m$ (i.e. as long as $\|q\|_{L^{\infty}<m / e}$; in the usual units $m / e$ should be replaced by $m c^{2} / e$ ). It is known [6] that if $\|q\|_{L^{\infty}}<m / e \sqrt{2}$ then $\mathscr{A}$ is a spectral operator.

We shall show that, as should be expected, $\|q\|_{L^{\infty}}$ is not so important; what matters is $\operatorname{diam} q=\operatorname{ess} \sup q-\operatorname{ess} \inf q$. If $\operatorname{diam} q<2 m / e$ then all the eigenvalues are real and semisimple; there are no eigenvalues in certain intervals; $\mathscr{A}$ is a selfadjoint operator in an appropriate scalar product. A real nonsemisimple eigenvalue can occur if $\operatorname{diam} q=2 \mathrm{~m} / \mathrm{e}$; however an eigenfunction must satisfy some additional, very restrictive stipulations, so this case is exceptional. For example if $A=0$ then all the eigenvalues are semisimple even if $\operatorname{diam} q=2 \mathrm{~m} / e$. If $A=0$ then $\mathscr{A}^{-1}$ is a real operator which is positivity preserving if $q$ is of constant sign (more generally if diam $q$ is small enough). In this case some interesting phenomena occur. Assume $q$ is negative and $\tilde{H}$ compact so the essential spectrum of $\mathscr{A}$ is $[-\infty,-m] \cup[m, \infty)$. Then all the discrete eigenvalues originate at $m$ and move to the left as $q$ decreases. The smallest eigenvalue is simple-it is a "ground state". When $\operatorname{diam} q=m / e$, there can be eigenvalues appearing at $-m$ and moving to the right as $\|q\|_{L^{\infty}}$ increases further. The largest of such eigenvalues is simple: it is another ground state. Thus we have (at most) two ground states.

The Klein paradox occurs when two ground states meet or when the right ground state hits $-m$ or the left ground state hits $m$. A precise statement concerning the multiplicity of such eigenvalues can be made. $\dagger$ 


\section{EIGENVALUES OF THE KLEIN-GORDON EQUATION}

Define $\mathscr{H}=\mathscr{D}\left(\tilde{H}^{1 / 2}\right) \times \mathscr{G} ; \quad \mathscr{H}$ is a Hilbert space in the norm $\|u\|=\left(\left\|\tilde{H}^{1 / 2} u_{1}\right\|^{2}\right.$ $\left.+\left\|u_{2}\right\|^{2}\right)^{1 / 2}$ where $u=\left[\begin{array}{ll}u_{1} & u_{2}\end{array}\right]$; we denote the norms in $\mathscr{H}$ and $\mathscr{G}$ by the same symbol, since it will always be clear to what space a vector belongs.

Define $\mathscr{A}$ on $\mathscr{D}(\mathscr{A})=\mathscr{D}(H) \times \mathscr{D}\left(\tilde{H}^{1 / 2}\right)$ by

$$
\mathscr{A}\left[\begin{array}{l}
u \\
v
\end{array}\right]=\left[\begin{array}{c}
v \\
H u+2 K v
\end{array}\right] \text {. }
$$

If $H$ is positive definite, the norm in $\mathscr{H}$ can be defined using $H$ instead of $\tilde{H} ; \mathscr{A}$ is selfadjoint in the corresponding scalar product.

If $\lambda \neq 0$ define

$H_{\lambda}=H+2 \lambda K-\lambda^{2}, K_{\lambda}=K-\lambda, \mathscr{S}_{\lambda}=\left[\begin{array}{cc}I & 0 \\ -\lambda & I\end{array}\right], \quad \mathscr{A}_{\lambda}=\left[\begin{array}{cc}0 & I \\ H_{\lambda} & 2 K_{\lambda}\end{array}\right] \quad$ on $\quad \mathscr{D}(\mathscr{A} \lambda)=\mathscr{D}(\mathscr{A})$.

Then $\mathscr{A}_{\lambda}=\mathscr{S}_{\lambda} \mathscr{A} \mathscr{S}_{\lambda}^{-1}-\lambda I$. Thus $\mu$ is an eigenvalue of $\mathscr{A}$ if and only if $\mu-\lambda$ is an eigenvalue of $\mathscr{A}_{\lambda}$. This can be seen equivalently by setting $u(t)=e^{i \lambda t} v(t)$ in (3). Then $v(t)$ satisfies (3) with $K, H$ replaced by $K_{\lambda}, H_{\lambda}$. This translation of spectral parameter represents the arbitrariness of choosing the zero potential.

Our results are contained in the next two theorems.

Theorem A. Let $\min \sigma(K)=1 / e$ ess inf $q=k_{2}, \max \sigma(K)=1 / e$ ess $\sup q=k_{1}$.

(a) The set of all $\lambda \in R$ such that $H_{\lambda}$ is positive semidefinite is either empty or a closed interval $\left[\lambda_{-}, \lambda_{+}\right]$. If the latter is the case, then $H_{\lambda_{-}}$and $H_{\lambda_{+}}$are semidefinite, $H_{\lambda}$ is positive definite if $\lambda_{-}<\lambda^{2} \lambda_{+}$and indefinite if $\lambda<\lambda_{-}$or $\lambda>\lambda_{+}$.

(b) There are no eigenvalues in $\left(\lambda_{-}, \lambda_{+}\right)$if $\lambda_{-}<\lambda_{+}$.

(c) If $k_{1}-k_{2} \leqq 2 m$ then $\lambda_{-}<k_{1}-m \leqq k_{2}+m<\lambda_{+}$and all the eigenvalues are real and semisimple.

(d) If $\lambda_{-}<\lambda_{+}$then $k_{2}>\lambda_{-}$implies $\lambda_{+} \geqq m, k_{1}<\lambda_{+}$implies $\lambda_{-} \leqq-m$.

(e) All the nonreal and nonsemisimple eigenvalues are contained in $\left\{z \in C: k_{2} \leqq \operatorname{Re} z \leqq k_{1},|z| \leqq m\right\}$.

Theorem B. Assume additionally $A=0$.

If $\lambda_{-}<\lambda_{+}$then $\lambda_{-}, \lambda_{+}$can be only simple eigenvalues. If $\lambda_{-}=\lambda_{+}=\tilde{\lambda}$ then $k_{2} \leqq \tilde{\lambda} \leqq k_{1},|\lambda| \leqq m$. If $\tilde{\lambda}$ is an eigenvalue, then $k_{2}<\tilde{\lambda}<k_{1}$ and it is an eigenvalue of geometric multiplicity one and algebraic multiplicity one or two. If

$$
q^{2} \text { is } \Delta^{2} \text {-compact }
$$

and $\lambda_{-}>-m\left(\lambda_{+}<m\right)$ then $\lambda_{-}$(respectively $\left.\lambda_{+}\right)$is an eigenvalue. If $\lambda_{-}=\lambda_{+}=\tilde{\lambda}$ then $\tilde{\lambda}$ is an eigenvalue (even if $|\lambda|=m$ ). If $|\lambda|<m$ the algebraic multiplicity of $\tilde{\lambda}$ is two. 
Proof of Theorem A. For $u \in \mathscr{G}$ with $\|u\|=1$ denote $p_{u}(\lambda)=-\left(H_{\lambda} u \mid u\right)=\lambda^{2}$ $-2 \lambda(K u \mid u)-(H u \mid u)$. If $p_{u}(\lambda)$ has real zeros denote the smaller by $\lambda_{-}(u)$, the larger by $\lambda_{+}(u)$. If $\lambda_{0}$ is an eigenvalue and $u_{0}$ an eigenfunction then $p_{u_{0}}\left(\lambda_{0}\right)=0$.

Now define

$$
\lambda_{+}=\inf _{\|u\|=1} \lambda_{+}(u), \quad \lambda_{-}=\sup _{\|u\|=1} \lambda_{-}(u)
$$

Since $H$ is unbounded, $p_{u}$. must have real zeros for some $u$, so $\lambda_{+}$and $\lambda_{-}$are well defined. Assume $\lambda_{-} \leqq \lambda_{+}$and let $\lambda_{-} \leqq \lambda \leqq \lambda_{+}$. For any $u \in \mathscr{G}$ with $\|u\|=1$ we find

$$
\left(H_{\lambda} u \mid u\right)=-p_{u}(\lambda)=\left(\lambda_{+}(u)-\lambda\right)\left(\lambda_{-}-\lambda_{-}(u)\right) \geqq\left(\lambda_{+}-\lambda\right)\left(\lambda_{-} \lambda_{-}\right) \geqq 0 .
$$

This means $\left(H_{\lambda} u \mid u\right) \geqq\left(\lambda_{+}-\lambda\right)\left(\lambda_{-} \lambda_{-}\right)\|u\|^{2}$ for every $u \in \mathscr{G}$. We conclude that $H_{\lambda}$ is positive definite if $\lambda \in\left(\lambda_{-}, \lambda_{+}\right)$. If $\lambda>\lambda_{+}$or $\lambda<\lambda_{-}$then there exists $u$ such that $p_{u}(\lambda)>0$ (this is the case as soon as $\lambda>\lambda_{+}(u)$ or $\lambda<\lambda_{-}(u)$ ), hence $\left(H_{\lambda} u \mid u\right)<0$ so $H_{\lambda}$ is indefinite. Now $H_{\lambda}$ is analytic in $\lambda$ and therefore $H_{\lambda}$ and $H_{\lambda+}$ are semidefinite. This proves (a); part (b) follows from (a).

Now let $\lambda$ be a nonsemisimple eigenvalue. Then we can find $u, v \in \mathscr{G}$ with $\|u\|$ $=1, \quad H_{\lambda} u=0, \quad H_{\lambda} v=2(K-\lambda) u$, hence $p_{u}(\lambda)=0$ and $((K-\lambda) u \mid u)=\frac{1}{2}\left(H_{\lambda} v \mid u\right)=0$. Since $p_{u}^{\prime}(\lambda)=2((\lambda-K) u \mid u)$, it follows that $p_{u}^{\prime}(\lambda)=0$. This implies $\lambda_{-}(u)=\lambda_{+}(u)$ so the discriminant of $p_{u}$ is zero. If $u$ is an eigenfunction of a nonreal eigenvalue then evidently $p_{u}$ has no real zeros, hence its discriminant is negative.

From this it follows that an eigenvalue $\lambda$ is real and semisimple if and only if the discriminant of $p_{u}$ is positive for every eigenfunction $u$ with $\|u\|=1$. The discriminant is

$$
(H u \mid u)+(K u \mid u)^{2}=(\tilde{H} u \mid u)^{2}-q_{K}(u) \quad \text { where } \quad q_{K}(u)=\|K u\|^{2}-(K u \mid u)^{2}
$$

Evidently $q_{K}(u) \leqq\|K\|^{2} ;$ if $k_{1}=-k_{2}$ the equality holds if and only if $\|K u\|=\|K\|$, $(K u \mid u)=0$. This is possible if and only if

$$
u=u_{1}+u_{2}, \quad\left\|u_{1}\right\|=\left\|u_{2}\right\|=\frac{1}{\sqrt{2}}, \quad K u_{1}=\|K\| u_{1}, \quad K u_{2}=-\|K\| u_{2} .
$$

Because of $q_{K+\lambda}=q_{K}$ we conclude that for arbitrary $K\|K u\|^{2}-(K u \mid u)^{2} \leqq \frac{1}{4}\left(k_{1}-k_{2}\right)^{2}$ for all $u$ with $\|u\|=1$; the equality holds if and only if $\tilde{u}=u_{1}+u_{2},\left\|u_{1}\right\|=\left\|u_{2}\right\|, K u_{i}$ $=k_{i} u_{i}, \quad i=1,2$. Therefore the discriminant is strictly positive if $k_{1}-k_{2}<2 m$; it is nonnegative if $k_{1}-k_{2}=2 m$; if there is an eigenfunction $\tilde{u}$ such that $p_{\tilde{u}}$ has double zero then this zero is necessarily equal to $(K \tilde{u} \mid \tilde{u})=\left(k_{1}+k_{2}\right) / 2$. In this case $\left(K-\left(k_{1}+k_{2}\right) / 2\right)^{2} \tilde{u}=\frac{1}{4}\left(k_{1}-k_{2}\right)^{2} \tilde{u}$, so

$$
\tilde{H} u=\left(K-k_{0}\right)^{2} u=m^{2} u, u=u_{1}+u_{2},\left\|u_{1}\right\|=\left\|u_{2}\right\|, K u_{i}=k_{i} u_{i}, i=1,2
$$

holds for some $u \neq 0$ and $k_{0}=\left(k_{1}+k_{2}\right) / 2$.

If $\|K-\lambda\|<m$ then $\dot{H}_{\lambda}=\tilde{H}-(K-\lambda)^{2}$ is positive definite; this is the case if $k_{1}$ $-m<\lambda<k_{2}+m$. This is possible only if $k_{1}-k_{2}<2 m$; if this is the case and $k_{1}-m$ is an eigenvalue, then obviously $\lambda_{-}=k_{1}-m$ and $\tilde{H} u=m^{2} u=\left(K-k_{1}+m\right)^{2} u$ for any 
eigenfunction $u$. Since $2 m-k_{1}+K \geqq 2 m-k_{1}+k_{2}>0$ we conclude that in this case there is $u \neq 0$ such that

$$
\tilde{H} u=m^{2} u, K u=k_{1} u
$$

In the same way, if $k_{2}+m$ is an eigenvalue, then there is $u \neq 0$ such that

$$
\tilde{H} u=m^{2} u, K u=k_{2} u
$$

Now by Kato's inequality $([7, \mathrm{II}])\left(\tilde{H}-m^{2}\right) u=0$ implies

$$
0=\left(\left(\tilde{H}-m^{2}\right) u \mid u\right)=\sum_{j}\left\|\left(\frac{\partial}{\partial x_{j}}-i e A_{j}\right) u\right\|^{2} \geqq \sum_{j}\left\|\frac{\partial}{\partial x_{j}}|u|\right\|^{2}
$$

hence $|u|$ is a constant, so $u=0$. Thus (6a), (6b) and (6c) are impossible and the sharp inequalities in (c) are proved.

Part (e) is proved in [6], and one part of (d) follows from the next lemma; the other part is proved in the same way.

Lemma. Let $\lambda \in\left(\lambda_{-}, \lambda_{+}\right)$and $K \geqq \lambda$. Then $\lambda_{+} \geqq m$. Moreover all the eigenvalues in $(-m, m)$ are nondecreasing functions of $K$ as long as $K \geqq \lambda>\lambda_{-}\left(\lambda_{-}\right.$depends on $\left.K\right)$.

Proof. Without loss of generality (translating the spectral parameter) we can assume $\lambda=0$. Define

$$
\begin{gathered}
H(\mu)=\tilde{H}-\mu^{2} K^{2},(u \mid v)_{\mu}=\left(H(\mu)^{1 / 2} u_{1} \mid H(\mu)^{1 / 2} v_{1}\right)+\left(u_{2} \mid v_{2}\right), \\
\mathscr{H}(\mu)=\left(\mathscr{H},(\mid)_{\mu}\right), \mathscr{A}(\mu)=\left[\begin{array}{cc}
0 & I \\
H(\mu) & 2 \mu K
\end{array}\right] \text { on } \mathscr{D}(\mathscr{A}) .
\end{gathered}
$$

Then

$$
\mathscr{A}(\mu)^{-1}=\left[\begin{array}{cc}
-2 \mu H(\mu)^{-1} K & H(\mu)^{-1} \\
I & 0
\end{array}\right]
$$

and

$$
f_{u}(\mu)=\frac{\left(\mathscr{A}(\mu)^{-1} u \mid u\right)_{\mu}}{2(u \mid u)_{\mu}}=\frac{\operatorname{Re}\left(u_{1} \mid u_{2}\right)-\mu\left(K u_{1} \mid u_{1}\right)}{\left(\tilde{H} u_{1} \mid u_{1}\right)+\left\|u_{2}\right\|^{2}-\mu^{2}\left\|K u_{1}\right\|^{2}}
$$

We shall prove that $f_{u}$ is monotone decreasing for all $u \in \mathscr{H}$ in $[0, m /\|K\|]$. By the min-max theorem this means that $\lambda_{+}(\mu) \geqq m$ for all $\mu$ in this interval (where $\lambda_{+}(\mu)$ is $\lambda_{+}$associated with $\mathscr{A}(\mu)$ ). Assume $K^{\prime} \geqq K>0, \tilde{H}-K^{\prime 2}>0$ (implying $\lambda_{-}^{\prime}<0$ ). Define $\mathscr{A}^{\prime},(\mid)^{\prime}$ using $K^{\prime}$ and let $f_{u}\left(K^{\prime}\right)=\left(\mathscr{A}^{-1} u \mid u\right) / 2(u \mid u), f_{u}\left(K^{\prime}\right)=\left(\mathscr{A}^{\prime-1} u \mid u\right)^{\prime} / 2(u \mid u)^{\prime}$. Since $\sigma_{p}\left(A^{\prime}\right) \cap(-m, m) \subset(-m, 0)$ it suffices to show $f_{u}(K)<0$ implies $f_{u}\left(K^{\prime}\right)<0$ (by the minmax theorem, it follows that the spectrum of $\mathscr{A}^{\prime-1}$ is to the left of the spectrum of 
$\left.\mathscr{A}^{-1}\right)$. This is evident since $f_{u}(K)<0$ implies

$$
\left|f_{u}(K)\right|=\frac{\left(K u_{1} \mid u_{1}\right)-\operatorname{Re}\left(u_{1} \mid u_{2}\right)}{\left(\tilde{H} u_{1} \mid u_{1}\right)+\left\|u_{2}\right\|^{2}-\left\|K u_{1}\right\|^{2}}
$$

and the denumerator is increasing while the denominator is decreasing with $K$.

To prove that $f_{u}(\mu)$ is monotone denote $\operatorname{Re}\left(u_{1} \mid u_{2}\right)=a,\left(K u_{1} \mid u_{1}\right)=b,\left(\tilde{H} u_{1} \mid u_{1}\right)$ $+\left\|u_{2}\right\|^{2}=c, \quad\left\|K u_{1}\right\|^{2}=d$. Then $f_{u}(\mu)=(a-b \mu) /\left(c-d \mu^{2}\right), \quad f_{u}^{\prime}(\mu)=-h(\mu) /\left(c-d \mu^{2}\right)^{2}$ with $h(\mu)=b d \mu^{2}-2 a d \mu+b c$. If $u$ is such that $a<0$ then $h(\mu)>0$ for all $\mu$ and $f_{u}$ is decreasing on $[0, \infty)$. If $a>0$ then $f_{u}$ is decreasing on $\left[0, \mu_{1}(u)\right]$ where $\mu_{1}(u)=$ $a d-\sqrt{ }\left(a^{2} d^{2}-b^{2} d c\right) / b d$ is the smaller root of $h(\mu)=0$. Now

$$
\begin{gathered}
\mu_{1}(u)=\frac{b c}{a d+\sqrt{\left(a^{2} d^{2}-b^{2} d c\right)} \geqq \frac{b c}{2 a d},} \\
c \geqq m^{2}\left\|u_{1}\right\|^{2}+\left\|u_{2}\right\|^{2} \geqq 2 m\left\|u_{1}\right\|\left\|u_{2}\right\| \geqq 2 m a, \\
d=\left\|K^{1 / 2} K^{1 / 2} u_{1}\right\|^{2} \leqq\left\|K^{1 / 2}\right\|^{2}\left\|K^{1 / 2} u_{1}\right\|^{2}=b\|K\|,
\end{gathered}
$$

hence $b c / 2 a d \geqq m /\|K\|$, so $f_{u}$ is decreasing in $[0, m /\|K\|]$ for all $u$.

\section{Remarks}

1. If

$$
q^{2} \text { is } \tilde{H}^{2} \text { compact }
$$

then $K$ and $K^{2}$ are $\tilde{H}^{2}$-compact, so $\sigma_{e}\left(H_{\lambda}\right)=\sigma_{e}\left(H-\lambda^{2}\right)([10])$, therefore $\sigma_{e}\left(H_{\lambda}\right)=\left[m^{2}\right.$ $\left.-\lambda^{2}, \infty\right)$. It follows that $(-m, m) \cap \sigma(\mathscr{A})$ consists only of eigenvalues which are increasing (respectively decreasing) functions of $q$ as long as $q$ is positive (respectively negative) and small enough; if $\lambda_{+}<m$ (or $\lambda_{-}>-m$ ) then $\lambda_{+}$ (respectively $\lambda_{-}$) is an eigenvalue.

2. If $A=0$, the conclusion that (6) has no solutions follows also from the unique continuation theorem except in the trivial case when $q$ is a constant or a step function attaining only two values.

3. The estimates in (c) can easily be strengthened. Note that $H_{\lambda}=\tilde{H}^{1 / 2}(I$ $\left.-X_{\lambda}^{*} X_{\lambda}\right) \widetilde{H}^{1 / 2}$ where $X_{\lambda}=K_{\lambda} \tilde{H}^{-1 / 2}$. Hence if $\left\|X_{\lambda}\right\|<1$ then $\lambda \in\left(\lambda_{-}, \lambda_{+}\right)$. Now Kato's inequality implies that a sufficient condition for $\left\|X_{\lambda}\right\|<1$ is $\left\|K_{\lambda} f\right\|^{2}<\|\nabla|f|\|^{2}$ $+m^{2}\|f\|^{2}$ for every $f \in H^{1}\left(R^{n}\right)$. Now $\|\nabla|f|\| \geqq\|f / 2 r\|$, so a sufficient condition is $(K$ $-\lambda)^{2}<m^{2}+1 / 4 r^{2}$, i.e.

$$
\lambda-\int\left(m^{2}+\frac{1}{4|x|^{2}}\right)<q(x)<\lambda+\int\left(m^{2}+\frac{1}{4|x|^{2}}\right) .
$$


In other words, if $q_{1}(x)=q(x)-\sqrt{ }\left(m^{2}+1 / 4|x|^{2}\right), \quad q_{2}(x)=q(x)+\sqrt{ }\left(m^{2}+1 / 4|x|^{2}\right)$ then $\operatorname{ess} \sup q_{1} \geqq \lambda_{-}$, ess inf $q_{2} \leqq \lambda_{+}$.

4. If $\lambda_{-}=\lambda_{+}=\lambda$ and if $\tilde{\lambda}$ is an eigenvalue, it cannot have a Jordan chain of length more than two. To prove this, we can again assume $\lambda=0$. Suppose $\mathscr{A}_{1}=0$, $\mathscr{A} e_{i}=e_{i-1}, i=2,3$ where $e_{i}=\left[x_{i} y_{i}\right]^{T}, i=1,2,3$. We immediately find $y_{1}=0, y_{i}=x_{i-1}$, $i=2,3$ and $H x_{1}=0, H x_{2}+2 K x_{1}=0, H x_{3}+2 K x_{2}=x_{1}$. Multiply the second equation by $x_{2}$, the third by $x_{1}$. We find

$$
2\left(K x_{1} \mid x_{2}\right)=-\left(H x_{2} \mid x_{2}\right), 2\left(K x_{2} \mid x_{1}\right)=\left\|x_{1}\right\|^{2},
$$

i.e. $\left\|x_{1}\right\|^{2}=-\left(H x_{2} \mid x_{2}\right)$. Since $H$ is semidefinite we have $x_{1}=0$, so $e_{1}=0$ and this is a contradiction.

Proof of Theorem B. If $\lambda_{-}$is an eigenvalue, the corresponding eigenfunction of $H_{\lambda_{-}}$is a ground state of a Schrödinger operator (note that $(H+2 \lambda K+\alpha)^{-1}$ is positivity improving for appropriate $\alpha$; cf. [7, IV]); therefore it is positive and the eigenvalue is simple.

Note that.(5) coincides with (7) if $A=0$ so we can use Remark 1 above to prove that if $\lambda_{-}>-m$ then it is a simple eigenvalue. All that is left to prove are the two last statements (the rest follows from Theorem $\mathrm{A}(\mathrm{e})$ and Remark 4 above). Assume $\tilde{\lambda}=-m\left(\tilde{\lambda}=m\right.$ is treated in the same way). Pick $\lambda_{0}>-m$ such that $H_{\lambda_{0}}$ is an indefinite operator with $0 \in \rho\left(H_{\lambda_{0}}\right)$ and with one dimensional negative part; define $\hat{H}(\mu)=\tilde{H}-\mu^{2}\left(K-\lambda_{0}\right)^{2}$ so that $\hat{H}(1)=H_{\lambda_{0}}$. Since $\hat{H}(\mu)$ is analytic in $\mu$, there is $\mu_{0}<1$ such that $0 \in \rho(\hat{H}(\mu))$ and $\hat{H}(\mu)$ has one dimensional negative part for all $\mu \in\left[\mu_{0}, 1\right]$. Define

$$
\begin{gathered}
{[u \mid v]_{\mu}=\left(\left.\operatorname{sgn} \hat{H}(\mu)|\hat{H}(\mu)|^{1 / 2} u_{1}|| \hat{H}(\mu)\right|^{1 / 2} v_{1}\right)+\left(u_{2} \mid v_{2}\right),} \\
\mathscr{H}(\mu)=\left(\mathscr{H},[\mid]_{\mu}\right), K(\mu)=\mu\left(K-\lambda_{0}\right), \\
\mathscr{A}(\mu)=\left[\begin{array}{cc}
0 & I \\
\hat{H}(\mu) & 2 K(\mu)
\end{array}\right] \text { on } \mathscr{D}(\mathscr{A}(\mu))=\mathscr{D}(\mathscr{A}) .
\end{gathered}
$$

Then $\mathscr{H}(\mu)$ is a Pontrjagin space of index $1([1]), \mathscr{A}(\mu)$ is selfadjoint in $\mathscr{H}(\mu)$. Therefore $\mathscr{A}(\mu)$ has a one dimensional invariant subspace $\mathscr{L}(\mu)$ such that $[\mid]_{\mu}$ is nonpositive on $\mathscr{L}(\mu)$. It is easy to see that this subspace is just the eigenspace associated with the simple eigenvalue $\lambda_{+}(\mu)$. Since $K(\mu) \leqq K(1)$ we have $\lambda_{+}(\mu)>\lambda_{-}(\mu)$ $=-m-\lambda_{0}$. Now $K(\mu)$ and $\hat{H}(\mu)^{-1}$ converge in norm to $K(1)$ and $\hat{H}(1)^{-1},[u \mid v]_{\mu}$ converges to $[u \mid v]_{1}$ (for all $u, v \in \mathscr{H}$ ) as $\mu \rightarrow 1$. The results of [2] imply that $\lim _{\mu \rightarrow 1} \lambda_{+}(\mu)=\hat{\lambda}$ is an eigenvalue of $\mathscr{A}(1)=\mathscr{A}_{\lambda_{0}}$. This eigenvalue cannot have a Jordan chain of length larger than three. Now $\lambda_{+}(\mu)$ is less than zero so $\hat{\lambda} \leqq 0$; in fact $\hat{\lambda}<0$ since $0 \in \rho\left(H_{\lambda_{0}}\right)$. If $[u \hat{\lambda} \hat{u}]^{T}$ is the eigenvector associated to $\hat{\lambda}$ then it is nonpositive in $\mathscr{H}(1)$, i.e. $\left(\left(H_{\lambda_{0}}+\hat{\lambda}^{2}\right) \hat{u} \mid \hat{u}\right)<0$. This immediately implies $\hat{\lambda}=\lambda_{+}(1)=-m-\lambda_{0}$. It follows that $-m$ is an eigenvalue of $\mathscr{A}$. 
Similarly, if $|\tilde{\lambda}|<m$ set $H(\mu)=\tilde{H}-\mu^{2}(K-\tilde{\lambda})^{2}$

$$
\mathscr{A}(\mu)=\left[\begin{array}{cc}
0 & I \\
H(\mu) & 2 \mu(K-\tilde{\lambda})
\end{array}\right] \text { on } \mathscr{D}(\mathscr{A})
$$

Then $\lim _{\mu \rightarrow 1} \lambda_{ \pm}(\mu)=0$. Since $\lambda_{ \pm}(\mu)$ are simple eigenvalues of $\mathscr{A}(\mu)$ by the above argument, it follows from the analytic perturbation theory ([4]) that zero is an eigenvalue of $\mathscr{A}(1)$ of algebraic multiplicity at least two. Now $\mathscr{A}(1)$ is similar to $\mathscr{A}-\tilde{\lambda}$, so the algebraic multiplicity of $\tilde{\lambda}$ is exactly two.

\section{Remarks}

1. The simplicity of eigenvalues $\lambda_{ \pm}$follows also by another argument. Let $\lambda \in\left(\lambda_{-}, \lambda_{+}\right)$. Then $H_{\lambda}^{-1}$ is positivity improving (Theorem XIII.45 in [7, IV]). Define $\mathscr{B}_{\lambda}$ as the extension of $\mathscr{A}_{\lambda}$ to $\mathscr{G}^{2}: \mathscr{D}\left(\mathscr{B}_{\lambda}\right)=\mathscr{D}\left(\mathscr{A}_{\lambda}\right), \mathscr{B}_{\lambda} u=\mathscr{A}_{\lambda} u, \mathscr{B}_{\lambda} u$ is regarded as a vector in $\mathscr{G}^{2}$. Then $\mathscr{B}_{\lambda}$ is closable and its closure is boundedly invertible. The inverse is

$$
\overline{\mathscr{B}}_{\lambda}^{-1}=\left[\begin{array}{cc}
-2 H_{\lambda}^{-1} K_{\lambda} & H_{\lambda}^{-1} \\
I & 0
\end{array}\right]
$$

Now

$$
\left[\begin{array}{cc}
0 & H_{\lambda}^{-1} \\
I & 0
\end{array}\right]
$$

is positivity improving, so there is a real number $v$ such that $v+\overline{\mathscr{B}}_{\lambda}^{-1}$ is positivity improving. This implies that if $\operatorname{spr} \overline{\mathscr{B}}_{\lambda}^{-1}$ is equal to the largest eigenvalue, then this eigenvalue is simple. It is easy to see that the eigenvalues of $\mathscr{A}_{\lambda}$ and $\overrightarrow{\mathscr{B}}_{\lambda}$ coincide. Therefore we conclude: if $\lambda_{+}$is an eigenvalue of $\mathscr{A}$, then $1 /\left(\lambda_{+}-\lambda\right)$ is the largest eigenvalue of $\overline{\mathscr{B}}_{\lambda}^{-1}$, hence it is simple. Since

$$
-\left[\begin{array}{cc}
-I & 0 \\
0 & I
\end{array}\right] \overline{\mathscr{B}}_{\lambda}^{-1}\left[\begin{array}{cc}
-I & 0 \\
0 & I
\end{array}\right]=\left[\begin{array}{cc}
2 H_{\lambda}^{-1} K_{\lambda} & H_{\lambda}^{-1} \\
I & 0
\end{array}\right]
$$

the same is true for the smallest eigenvalue of $\overline{\mathscr{B}}_{\lambda}^{-1}$.

2. If $\|q\|_{L^{\infty}}$ is small enough and $q$ tends to zero at infinity, then there are no eigenvalues ([5]).

3. Sufficient conditions for (5) can be found in [7, II] and [8].

4. If $A \neq 0$ and $\lambda_{+}$is a discrete eigenvalue, then $\lambda_{+}>\lambda_{+}^{0}$ where $\lambda_{+}^{0}$ is $\lambda_{+}$for the case $A=0$. In fact, assume $H_{\lambda+} u=0$, i.e.

$$
D^{2} u=\left(K-\lambda_{+}\right)^{2} u-m^{2} u \quad \text { where } \quad D^{2}=\sum_{j}\left(\frac{\partial}{\partial x_{j}}-i e A_{j}\right)^{2}
$$


By Kato's inequality (generalised to forms: [9]),

$$
\operatorname{Re}\left[\arg u D^{2} u\right]=\operatorname{Re}\left\{\left[\left(K-\lambda_{+}\right)^{2}-m^{2}\right] u \arg u\right\}=\left[\left(K-\lambda_{+}\right)^{2}-m^{2}\right]|u| \geqq-\Delta|u|
$$

in the distribution sense. It follows that $\left\|\operatorname{grad}\left|u\left\|^{2}-\right\| K\right| u \mid\right\|^{2}+2 \lambda_{+}(K|u||| u \mid)+$ $\left(m^{2}-\lambda_{+}^{2}\right)\||u|\|^{2} \leqq 0$, so $H_{\lambda_{0}}^{0}$ is not positive definite; hence $\lambda_{+}^{0}<\lambda_{+}$. Analogously $\lambda_{-}<\lambda_{-}^{0}$. In other words, the magnetic potentials postpone the occurrence of the Klein paradox.

5. If $|\lambda|=m$, then $\mathscr{A}$ is a spectral operator (the same conclusion trivially holds if $|\lambda|<m$ ): $\mathscr{A}=\mathscr{S}+\mathscr{N}$ where $\mathscr{S}$ is a spectral operator of scalar type and $\mathscr{N}=0$ (if the algebraic multiplicity of $\tilde{\lambda}$ is one) or $\mathscr{N}$ is an operator of rank one (if the algebraic multiplicity is two).

To prove this pick $\lambda_{0}$ such that $0 \in \rho\left(H_{\lambda_{0}}\right)$ and $H_{\lambda_{0}}$ has one dimensional negative part. Define

$$
[u \mid v]=(u \mid v)_{\mathscr{H}}+2 \lambda_{0}\left(K u_{1} \mid v_{1}\right)-\lambda_{0}\left[\left(u_{1} \mid v_{2}\right)+\left(u_{2} \mid v_{1}\right)\right]
$$

Then $\mathscr{K}=(\mathscr{H},[\mid])$ is a Pontrjagin space of index 1 . It is sufficient to prove that the principal subspace (i.e. the generalised eigenspace) $X$ associated to $\tilde{\lambda}$ is nondegenerate in $\mathscr{K}$; the construction and the facts we use can be found in [6] and its references). If $\operatorname{dim} X=1$ this is evident; then $\mathscr{A} x=\tilde{\lambda} x$ implies $[x \mid x]<0$. If $\operatorname{dim} X=2$ let $\mathscr{A} e=\tilde{\lambda} e, \mathscr{A} f=\tilde{\lambda} f+e$. We find

$$
e=\left[\begin{array}{c}
x \\
\tilde{\lambda} x
\end{array}\right], \quad f=\left[\begin{array}{c}
y \\
\tilde{\lambda} y+x
\end{array}\right] \text { where } H_{\lambda} x=0, H_{\lambda} y+2 K_{\lambda} x=0 .
$$

Now $[e \mid e]=0$ (this is a general fact);

$$
[e \mid f]=\left(\tilde{\lambda}-\lambda_{0}\right)\left[\|x\|^{2}-2\left(K_{\tilde{\lambda}} x \mid y\right)\right]=\left(\tilde{\lambda}-\lambda_{0}\right)\left[\|x\|^{2}+\left(H_{x} y \mid y\right)\right] \neq 0,
$$

so $X$ is nondegenerate.

6. From Theorems $\mathrm{A}$ and $\mathrm{B}$ we have the following picture: assume $A=0, q$ is positive and zero at infinity. Consider $\mu q$ and increase $\mu$ from zero to infinity. As long as $\mu$ is sufficiently small, there are no eigenvalues. For some $\mu_{0}$ a negative eigenvalue appears at $-m$ and moves to the right as $\mu$ increases. After some time it is (possibly) followed by another eigenvalue appearing in the same manner, etc. The largest eigenvalue $\lambda_{-}(\mu)$ remains simple. All the eigenvalues move to the right until $\lambda_{-}(\mu)$ becomes zero (this cannot happen as long as $\mu<\|q\|_{L^{\infty}} / e$ ). As $\mu$ increases further the eigenvalues are not monotone in $\mu$ any more; it is possible that eigenvalues emerge at $m$. If this is the case, the smallest of them, $\lambda_{+}(\mu)$, is simple. As $\mu$ increases, two cases are possible. In the first case $\lambda_{+}(\mu)$ and $\lambda_{-}(\mu)$ meet at $\tilde{\lambda}, \tilde{\lambda}$ is between $-m$ and $m$, to produce an eigenvalue of geometric multiplicity one and algebraic multiplicity two. If $\mu$ is increased further this eigenvalue splits into a nonreal pair, symmetric with respect to the real axis (this 
follows from the analytic perturbation theory ([4]); the author is indebted to Prof. K. Veselic for this remark).

In the second case $\lambda_{-}(\mu)$ hits $m\left(\lambda_{+}(\mu)\right.$ cannot hit $-m$ by (d) of Theorem A). In that case an eigenvalue of geometric multiplicity one and algebraic multiplicity one or two is produced.

The last statement is particularly interesting since it concerns an eigenvalue embedded in a continuous spectrum; such eigenvalues are usually difficult to handle. Thus the Klein paradox occurs for the first time either by a collision of simple eigenvalues $\lambda_{+}(\mu)$ and $\lambda_{-}(\mu)$ between $-m$ and $m$ or by the "negative" eigenvalue $\lambda_{-}(\mu)$ entering the positive essential spectrum at $m$.

\section{REFERENCES}

1. Bognár, J., Indefinite Inner Product Spaces (Springer, 1974).

2. Langer, H. and Najman, B., Perturbation theory of definitizable operators, $J . O p$. Theory, to appear.

3. Leinfelder, H. and Simader, C. G., Schrödinger operators with magnetic vector potential, Math. Z. 176 (1981), 1-20.

4. Kato, T., Perturbation Theory for Linear Operators (Springer, 1966).

5. NaJman, B., Wave operators and similarity for some matrix operators with applications, Glasnik Mat. 14 (34) (1979), 289-307.

6. Najman, B., Localization of the critical points of Klein-Gordon type operators, Math. Nachr. 99 (1980), 33-42.

7. ReED, M. and Simon, B., Methods of Modern Mathematical Physics, I-IV (Academic Press).

8. Schechter, M., Spectra of Partial Differential Operators (North-Holland, 1971).

9. Simon, B., An abstract Kato's inequality for generators of positivity preserving semigroups, Indiana Univ. J. 26 (1977), 1067-1073.

10. Weidmann, J., Spectral theory of partial differential operators, Proc. of Spectral Theory and Differential Equations (Lecture Notes in Mathematics 448, Springer, 1975).

11. Veselic, K., On the nonrelativistic limit of the bound states of the Klein-Gordonequation, J. Math. Anal. Appl., to appear.

UNIVERSITY OF ZAGREB

YugosLavia 